\title{
Escolha de parceiros afetivos: influência das seqüelas de fissura labiopalatal
}

\author{
Fernanda Renda Brasil \\ Liliam D'Aquino Tavano \\ Universidade de São Paulo, Bauru-SP, Brasil \\ Sandro Caramaschi \\ Olga Maria Piazentin Rolim Rodrigues \\ Universidade Estadual Paulista Júlio de Mesquita Filho, Bauru-SP, Brasil
}

\begin{abstract}
Resumo: Adolescentes, fissurados ou não, se vêem diante de sensações novas perante si mesmos e em relação ao sexo oposto durante a busca de parceiros afetivos, estabelecendo critérios para essa seleção. Este estudo objetivou verificar critérios que adolescentes fissurados estabelecem para selecionar parceiros afetivos e se esses diferem dos utilizados pelos seus pares, não fissurados. Participaram, respondendo a um questionário, 74 adolescentes, de 14 a 21 anos, de ambos os sexos, divididos em dois Grupos: Fissurados (GF) e Não Fissurados (GNF). Os resultados sugerem que não há diferença significativa entre os grupos considerando as características que valorizam em si e no outro para a escolha do parceiro afetivo. Verificou-se que o GNF valorizou mais o rosto do que o GF, apresentando correlação estatisticamente significativa. Tais resultados permitiram concluir que a fissura interfere na visão de si mesmo ou do outro na busca por parceiros afetivos.
\end{abstract}

Palavras-chave: Seleção de parceiros. Adolescente. Fissura labial. Fissura palatina.

\section{Choice of affective partners: influence of sequel of labiopalatal fissure sequela}

Abstract: Adolescents, fissured or not, experience new emotions regarding themselves and the opposite sex when they search for affective partners, establishing criteria for this selection. This study aims to know the criteria used by fissured adolescents in selecting affective partners and whether such criteria differ from those employed by their peers. A total of 74 adolescents, both genders, aged 14 to 21 years, answered a questionnaire and were divided in two groups: Fissured (FG) and Not Fissured (NFG). Results suggest there is no expressive difference between groups, considering the characteristics they value in themselves and in others, for choosing affective partners. It was verified that the NFG adolescents give more value to the face than do FG adolescents, presenting statically significant correlation. These results permitted to infer that the fissure partially interferes in the perception of oneself and of the other in the search for affective partners.

Keywords: Mate selection. Adolescent. Cleft lip. Cleft palate.

\section{Elección de parejas: influencia de las secuelas de fisura labiopalatina}

Resumen: Adolescentes, con fisuras o no, se deparan ante nuevas sensaciones frente a sí mismos y en relación con el sexo opuesto durante la busca de parejas, estableciendo criterio para esa elección. Este estudio tiene como objetivo verificar los criterios que los adolescentes con fisuras establecen para elegir parejas y si tales criterios difieren de los utilizados por sus parejas que no tiene fisuras. Participaron, contestando a un cuestionario, 74 adolescentes de 14 a 21 años, de ambos sexos, divididos en dos grupos: los con fisuras (GF) y de los sin fisuras (GNF). Los resultados sugieren que no hay diferencia significativa entre los grupos, considerando las características que valoran en sí y en el otro para la elección de parejas. Se verificó que el GNF valoró más la cara que el GF, presentando correlación estadísticamente significativa. Tales resultados permitieron concluir que la fisura interfiere en la visión de sí mismo o del otro en la búsqueda de la pareja.

Palabras clave: Elección de parejas. Adolescente. Fisura labial. Fisura palatina. 


\section{Introdução}

A adolescência é uma época de transições em que mudanças importantes acontecem tanto no aspecto físico como emocional do adolescente, englobando todo seu desenvolvimento. Contini (2002) concebe esta fase como uma etapa marcada por tormentos e conturbações vinculadas à emergência da sexualidade. Essa concepção foi reforçada por algumas abordagens psicanalíticas que a caracterizaram como uma etapa de confusões, estresse e luto, também causados pelos impulsos sexuais que emergem nessa fase do desenvolvimento. Este momento se mostra ambíguo, devido às aquisições e perdas, em que a elaboração do luto deve permitir preservar internamente a imagem de uma infância valorizada que, simultaneamente, sobrevive como processo de construção e é perdida como realidade passada.

As experiências sexuais nessa fase se mostram cruciais para o desenvolvimento adequado dos referenciais psicológicos. Experiências negativas podem produzir dificuldades na percepção e no relacionamento com o sexo oposto (Rodrigues, Brino $\&$ Williams, 2006). Bee (1996) e Contini (2002) sugerem que o início da adolescência é uma época dominada pela assimilação de novos conhecimentos, inclusive sobre si mesmo, enquanto o seu final é principalmente um momento de acomodação destes conteúdos assimilados.

$\mathrm{O}$ adolescente de 12 ou 13 anos assimila uma enorme quantidade de experiências físicas, sociais e intelectuais novas. Enquanto essa absorção está acontecendo, mas antes de ser elaborada, o jovem fica mais ou menos em um contínuo estado de desequilíbrio. Os antigos padrões e esquemas já não funcionam bem e, os novos, ainda não estão estabelecidos. É durante esse período inicial que o grupo de companheiros se torna importante (Bee, 1996).

Os adolescentes demonstram ainda o papel fundamental do diálogo em família para fazer as acomodações necessárias e estabelecem uma nova identidade, novos padrões de relacionamento social, papéis sociais e novos objetivos para o futuro (Pratta \& Santos, 2007). Essas mudanças significativas impulsionam o jovem à busca de independência, acarretando, quase que inevitavelmente, conflitos com os pais acerca dos limites estipulados por eles (Tiba,
2005; Pratta \& Santos, 2007). O desenvolvimento de autonomia e tomada de decisões fazem parte deste período, neste sentido as meninas apresentam maior nível de autonomia decisional, enquanto os meninos experimentam maiores conflitos (Celen, Cok \& Bosma, 2006).

De acordo Bee (1996), o aumento no índice de depressão e a queda na auto-estima observados no início da adolescência parecem estar ligados a esse excesso de novas exigências e mudanças. Mas, a maneira como cada jovem enfrenta as mudanças pode ser essencial para a diminuição do estresse e menor probabilidade de uma auto-estima diminuída.

Para Goffman (1988), a estigmatização é decorrente de uma sociedade que preconiza o modelo do ser ideal, agredindo, de certa forma, o diferente com olhares de curiosidade e perguntas especulatórias. Estudos vêm sendo realizados com adolescentes no intuito de quantificar os efeitos de características pessoais como dominância (Pellegrini \& Long, 2003), etnia (Duke, 2002), doenças crônicas (Erkolahti, Ilonen \& Saarijavi, 2003; Hintikka, Pelkonen, Hintikka, Laukkanen \& Lehtonen, 2002) e classes sociais (Gomes \& Caramaschi, 2007) na auto-imagem.

$\mathrm{O}$ adolescente com seqüela de fissura palatolabial, além das mudanças comuns nessa fase, parece ter que enfrentar uma outra questão que é defrontarse com a idealização do belo. É um fator culturalmente relevante nas relações interpessoais (Jones e cols., 2005), cujo poder social tem determinado a estigmatização do diferente (Amaral, 1995). O adolescente vivencia, então, a aceitação de sua imagem ainda em transformação pela ação dos hormônios e, também, as transformações cirúrgicas, fonéticas e odontológicas decorrentes de seu tratamento.

A formação de uma auto-imagem positiva pode ser prejudicada devido à ansiedade, medo e vergonha que ocorre nos contatos sociais, podendo aumentar, ainda mais, o desajustamento social, visto que o diferente ainda é objeto de discriminação e preconceito em nossa sociedade, tendo o significado muitas vezes de inferior (Bachega, 2002).

É nessa fase da vida que os adolescentes, com seqüela de fissura palato-labial ou não, se vêem diante de sensações novas perante si mesmos e em relação 
ao sexo oposto. É quando a maturidade genital o estimula para relacionar-se com o outro sexo, iniciando a busca do objeto de amor no mundo externo (Aberastury, 1990). Essa busca pode ter várias vertentes, mas a idealização nessa fase da vida é muito intensa, fazendo com que os jovens estabeleçam certos critérios para a seleção de seus parceiros afetivos (Lazar \& Guttmann, 2004; Shackelford, Schmitt \& Buss, 2005).

Pesquisas feitas com adultos jovens (Kenrick, Sadalla, Groth \& Trost, 1990; Li \& Kenrick, 2006) e de forma semelhante com adolescentes (Regan \& Joshi, 2003) evidenciaram que as características desejáveis num possível parceiro amoroso mudam de acordo com a finalidade do relacionamento, se for considerado de curto prazo (ficar, paquerar) ou de longo prazo (namorar, casar).

A beleza é um fator relevante na hora da paquera ou do flerte (Maner, 2003). O corpo é abordado em termos estéticos, é objeto de desejo e fonte de prazer (Chaves, 2001). A fase do flerte é muito importante para a formação do par romântico, pois leva as pessoas a se aproximarem e a se conhecerem. Nesta fase os parceiros sentem-se empolgados, alertas, vivos e atraentes (Davis, 1979). Silva (2001) refere que as pessoas apresentam sinais de que desejam a privacidade, impedindo a aproximação de terceiros. Para que haja flerte, primeiro a pessoa precisa eleger um parceiro. Para Silva (2001) existem três fatores que determinam a forma de proceder na hora da escolha de um parceiro amoroso, são eles: universais, culturais e idiossincráticos.

Os fatores universais são aqueles compartilhados por quase todos os seres humanos como, por exemplo, a valorização da juventude, os sinais de saúde e os aspectos sexuais (Shackelford, Schmitt $\&$ Buss, 2005). Os fatores culturais são aqueles que podem variar entre culturas e épocas diferentes, sendo influenciado pelo ambiente físico e cultural. Já os fatores idiossincráticos são aqueles que podem variar bastante de uma pessoa para outra (Silva, 2001). Para este autor, a seleção de parceiros segue também um conjunto de normas ou princípios, que ajudam a pessoa decidir as qualidades que querem e os defeitos que não querem no parceiro, facilitando assim as escolhas que acabam sendo feitas através de médias ponderadas.
De acordo com a Teoria da Dissonância Cognitiva proposta por Festinger (1962/1975), muitas vezes ao se enfrentar uma situação de decisão, se estabelece uma contraposição entre as possibilidades de escolha. A opção por uma delas normalmente desencadeia um mecanismo cognitivo de convencimento de que a decisão tomada foi a melhor possível, desvalorizando-se a opção preterida. Fisher (1995) em seu livro "Anatomia do amor" descreve os padrões de comportamento mais comuns no flerte e faz uma analogia com o desenvolvimento de outras espécies animais em sua evolução durante a conquista do parceiro. Refere que há um padrão universal de comportamentos no flerte, com traços tão característicos que podem ser considerados inatos, traços típicos que vêm se desenvolvendo por milhões de anos para demonstrar o interesse sexual. Outros autores argumentam que mulheres e homens utilizam táticas de flerte similares àquelas encontradas em outras espécies (Weisfeld \& Woodward, 2004).

Durante o flerte a comunicação não verbal é a mais observada. A linguagem do corpo em suas expressões é evidente, mesmo que inconscientes para o indivíduo. Segundo Davis (1979) uma reação emocional, como a atração sexual, provoca ligeiras alterações no corpo, predispondo a pessoa ao flerte. De certa forma essa predisposição corporal está ligada à tensão muscular, os músculos se retesam em resposta à atenção, de maneira que o corpo inteiro torna-se subitamente alerta. No rosto as linhas antes flácidas, deixam de sê-lo e até as olheiras tendem a desaparecer. O olhar fica mais intenso e a textura da pele se altera. Dentro dessa comunicação não verbal está um dos prováveis instrumentos mais importantes entre os seres humanos no momento da paquera - a linguagem do olhar. Este desperta uma parte primitiva do cérebro causando a reação de rejeição ou aceitação, imprescindível para a aproximação de pares (Fisher, 1995).

Diante desses conceitos, o presente estudo se propôs a estudar quais critérios os adolescentes portadores de seqüelas de fissura labiopalatal estabelecem para selecionar parceiros afetivos e, se esses diferem dos estabelecidos pelos pares da sua idade cronológica não portadores de fissura labiopalatal. Espera-se entender um pouco sobre a socialização destes indivíduos e a possível influência da fissura na sua auto-estima. 
Por uma questão de praticidade lingüística, os portadores de seqüelas de fissura labiopalatal serão denominados neste trabalho como "grupo de fissurados", embora tais indivíduos não apresentassem de fato fissura labiopalatal, uma vez que se encontravam em estágios variados de tratamento. Cabe lembrar que se trata de uma terminologia eminentemente técnica, portanto livre de qualquer conotação pejorativa acerca dos participantes. Por questão de simetria, o grupo de participantes não portadores de fissura labiopalatal será denominado, neste artigo, como "grupo de não fissurados".

O presente trabalho tem como objetivo verificar se há diferenças nos critérios e características físicas presentes na escolha de parceiros afetivos para adolescentes fissurados e não fissurados. Os objetivos específicos foram: (1) descrever os critérios de seleção de parceiros afetivos em adolescentes fissurados; (2) identificar se há diferença nos critérios de seleção de parceiros entre adolescentes fissurados e não fissurados; (3) descrever as características importantes para si e para o outro na seleção de parceiros afetivos e, (4) verificar se há correlação entre as características elegíveis como importantes para si e para o outro na seleção de parceiros, entre adolescentes fissurados e não fissurados.

\section{Método}

\section{Participantes}

Participaram do estudo 74 adolescentes, na faixa etária de 14 a 21 anos, de ambos os sexos. O Grupo de Fissurados (GF) era composto por 37 adolescentes com seqüelas de fissura labiopalatal, sendo $51 \%$ mulheres e $49 \%$ homens, provenientes de várias cidades do Brasil. Dos participantes deste grupo, 68\% deles se encontram na faixa etária de 16 a 19 anos e 11 meses, sendo que $81 \%$ freqüentavam o ensino médio. Todos os participantes desse Grupo são pacientes do Hospital de Reabilitação de Anomalias Craniofaciais da Universidade de São Paulo-Bauru, que tiveram algum tipo de fissura labiopalatal e estão, ainda, em tratamento para reabilitação estética e/ou funcional.

No Grupo de Não Fissurados (GNF) participaram 37 adolescentes, sendo $62 \%$ mulheres e $38 \%$ homens, com a mesma faixa etária, alunos de uma escola pública de um município no interior do estado de São Paulo. Dos participantes, 73\% deles se encontram na faixa etária de 16 a 19 anos, sendo que todos freqüentavam o ensino médio. Observa-se que os participantes do Grupo GF apresentam atraso na escolaridade quando comparados ao Grupo GNF. Considerando-se que a idade média de conclusão no ensino médio é de 18 anos, no Grupo GF 16\% dos participantes estão acima de 20 anos, enquanto que no Grupo GNF, apenas 5\% deles estão nesta condição. Um outro dado é a presença de 19\% dos participantes do Grupo GF, ainda no ensino fundamental.

\section{Materiais}

Para a realização da pesquisa foi utilizado um questionário elaborado especificamente para este estudo, contendo espaço para os dados pessoais e com sete questões objetivas, abertas e fechadas, pertinentes a essa investigação. Nas questões 1 e 5 havia dez aspectos (características que poderiam determinar a escolha de pessoas para seu relacionamento afetivo ou que valorizam em si mesmo) que deveriam ser enumerados de 1 a 10 de acordo com a importância dada a ele, sendo 1 o menos importante. As questões 2 a 4 eram de múltipla escolha, onde o participante deveria assinalar uma das respostas que versavam sobre características gerais do rosto ou do corpo que apreciavam no outro. Nas questões 6 e 7 deveria discorrer sobre o que faz para paquerar e o que espera que o outro faça se quiser paquerá-lo.

Utilizou-se também o Termo de Consentimento Livre e Esclarecido e uma carta de informação ao sujeito sobre a pesquisa. Precedendo a execução da coleta de dados o projeto foi enviado ao Comitê de Ética do Hospital de Reabilitação de Anomalias Crânio Faciais (USP-Bauru), tendo sido aprovado.

\section{Procedimento}

A coleta de dados para o grupo GF foi realizada durante o atendimento ambulatorial, nos meses de agosto a novembro, a partir da identificação dos pacientes que se enquadravam no perfil estipulado para a pesquisa, em termos de idade (14 a 21 anos) e sem síndromes associadas. Cada paciente foi, então, convidado a participar do projeto, em horário interconsulta. Com o aceite, informações sobre o 
projeto foram fornecidas. Os que tinham idade acima de 18 anos, assinaram o Termo de Consentimento Livre e Esclarecido. Os que tinham idade inferior, o termo foi assinado pelo seu responsável legal. Em seguida, individualmente, responderam ao questionário.A aplicação do questionário com os adolescentes que têm seqüela de fissura lábio palatina foi realizada individualmente dentro do ambulatório do Hospital de Reabilitação de Anomalias Craniofaciais.

Para o grupo GNF, após a autorização da direção de uma escola de ensino médio, pública, contendo salas de aula que reuniam adolescentes da faixa etária requerida (14 a 21 anos), os alunos foram convidados a participar da pesquisa. Os que aceitaram foram levados para uma outra sala onde se explicou o projeto em linhas gerais. Para os alunos menores de 18 anos foram encaminhados os Termos de Consentimento Livre e Esclarecido para prévia autorização dos responsáveis legais, já os maiores de 18 anos assinaram o Termo. De posse desse documento, iniciou-se a aplicação do questionário em sala coletiva, nas dependências da escola, garantindo a privacidade dos participantes, com a pesquisadora presente todo o tempo para esclarecimento das dúvidas que emergissem.

A diferença na aplicação dos Grupos GF e GNF se deve à presença de adolescentes no HRAC-USP (Hospital de Reabilitação de Anomalias CraniofaciaisUSP) diariamente, porém não em número que justificasse uma aplicação coletiva. Por outro lado, o objetivo da sua presença no local não era a pesquisa, mas sim o acompanhamento do seu processo de reabilitação, o que possibilitou sua participação apenas em horários de intervalo das consultas ambulatoriais previamente agendadas, como parte do seu tratamento. Considerando a autonomia dos participantes do Grupo GNF em responder as questões na própria escola, optou-se pela aplicação coletiva.

\section{Análise de dados}

A análise dos dados obtidos foi realizada através de estatística não-paramétrica, utilizando-se o aplicativo Biostat (4.0). As comparações entre grupos acerca das características consideradas relevantes foram feitas através do Teste de MannWhitney, Índice de Correlação de Spearman e Teste

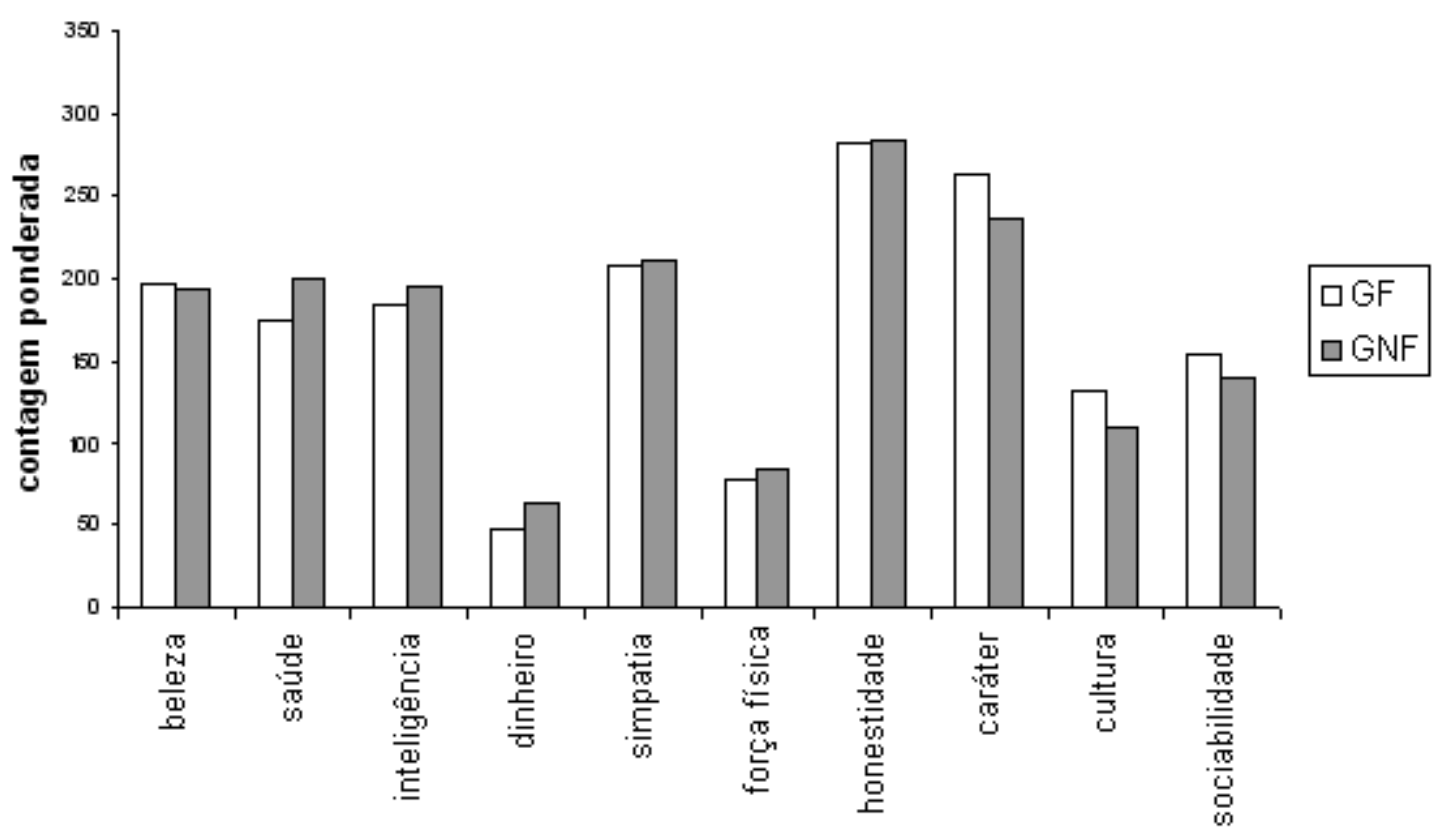

Figura 1. Caracteristicas do outro que determinam a escolha de pessoas para o relacionamento afetivo, para os participantes dos Grupos Fissurados (GF) e Não Fissurados (GNF). 
de Qui-quadrado. A categorização das questões abertas foi feita através da Análise de Conteúdo.

\section{Resultados e discussão}

O objetivo deste trabalho foi o de verificar se há diferenças nos critérios de escolha de parceiros afetivos no que diz respeito às características consideradas importantes por adolescentes fissurados e não fissurados.

Na Questão 1 solicitava-se que o sujeito classificasse de 1 a 10 (sendo 1 o menos valorizado), as características que determinavam a escolha de um parceiro para relacionamento afetivo a partir de uma lista previamente estabelecida. Diante das respostas foi feita a quantificação ponderada das mesmas. A Figura 1 apresenta os resultados obtidos quanto às características que determinavam a escolha do parceiro para o relacionamento afetivo, para os dois grupos.

Frente às respostas obtidas, pode-se perceber que não houve diferença significativa entre os grupos pelo Teste de Mann-Whitney ( $\mathrm{U}=30,5 ; p=0,1405)$.
As características honestidade e caráter foram as mais valorizadas e dinheiro e força física as consideradas menos importantes.

Para a questão relacionada aos aspectos importantes neles mesmos, foi solicitado que também classificassem as características apresentadas de 1 a 10 (sendo 1 o que menos valoriza). As respostas dadas pelos sujeitos estão representadas na Figura 2.

Analisando-se as características consideradas mais importantes neles próprios, observa-se que quanto às menos valorizadas, dinheiro e força física, houve acordo entre os grupos e entre as mais valorizadas, honestidade e caráter, também. O Teste de Mann-Whitney aplicado entre os grupos não evidenciou diferença significativa $(\mathrm{U}=31,0 ; p=0,1509)$.

Utilizou-se a correlação de Spearman para avaliar se o que eles dizem que é importante no outro é considerado importante nele mesmo, comparando, para cada grupo as respostas das duas questões. Observou-se uma correlação significativa para o GF ( $\mathrm{rs}=0,8667 ; p=0,0012)$ e para o GNF (rs $=0,9423$; $p=0,0000)$. Isso evidencia o que Silva (2001) refere

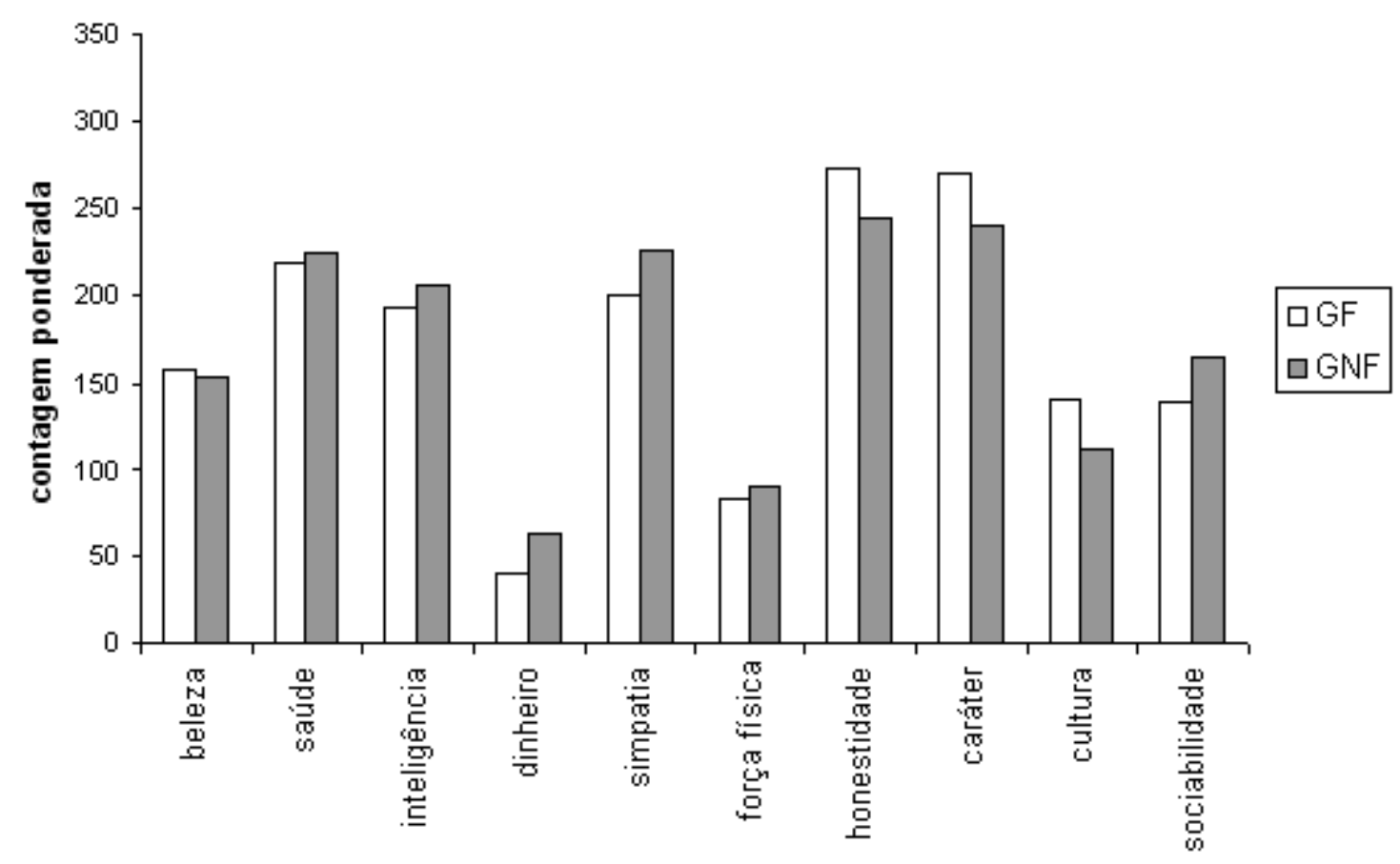

Figura 2. Características consideradas importantes em si mesmos para o relacionamento afetivo, para os participantes dos Grupos Fissurados (GF) e Não Fissurados (GNF). 
como um dos princípios mais adotados na seleção de parceiros amorosos, o princípio da homogamia, que afirma que os relacionamentos têm chance maior de "dar certo" quando os parceiros são semelhantes entre si. Tais resultados coincidem com os obtidos por Buston e Emlen (2003), onde as características desejadas em um parceiro de longo prazo coincidem com os atributos do próprio participante da pesquisa. Outros trabalhos (Michael, Gagnon, Laumann \& Kolata, 1995; Blackwell \& Lichter, 2004) corroboraram os resultados relativos à homogamia na seleção de parceiros.

Inquiridos sobre que aspectos físicos de uma pessoa são considerados importantes para um relacionamento afetivo, observou-se que os participantes do Grupo GF (55\%) relataram considerar aspectos físicos menos importantes do que o Grupo GNF (39\%). Porém, os que optaram por descrever qual aspecto físico mais valorizam o Grupo GNF (45\%) indicou o rosto muito mais do que o Grupo GF (24\%). Ainda que a diferença seja pequena entre os grupos para a indicação do corpo, $21 \%$ para o Grupo GF e $16 \%$ para o Grupo GNF, pode-se dizer que o GF valoriza mais o corpo.
Pode-se averiguar, pelo teste de qui-quadrado, uma diferença significativa de valorização dos aspectos físicos entre os dois grupos $\left(\mathrm{X}^{2}=9,79\right.$; $p=0,0075)$, tendo o GNF valorizado muito mais o rosto do que o GF. Tais dados parecem indicar uma possível influência da fissura.

Segundo a Teoria da Dissonância Cognitiva (Festinger, 1962/1975), as pessoas tendem a valorizar aspectos associados a si próprios e concomitantemente tendem a desvalorizar características que não possuem. Dessa forma, os adolescentes fissurados tendem a dizer (mais que os não fissurados) que aspectos corporais não são importantes na seleção de parceiros, bem como o rosto em particular. Tal mecanismo de defesa cognitiva serve como uma forma de sustentar uma auto-estima elevada, apesar de todas as evidências sociais de valorização da beleza física (Figura 3).

Dentre os aspectos do rosto, perguntou-se aos participantes qual ou quais consideravam importantes para o seu relacionamento afetivo. Os dois grupos valorizam mais os olhos, sendo 34\% para o Grupo GF e $32 \%$ do GNF. Em relação ao aspecto menos valorizado também houve concordância entre os

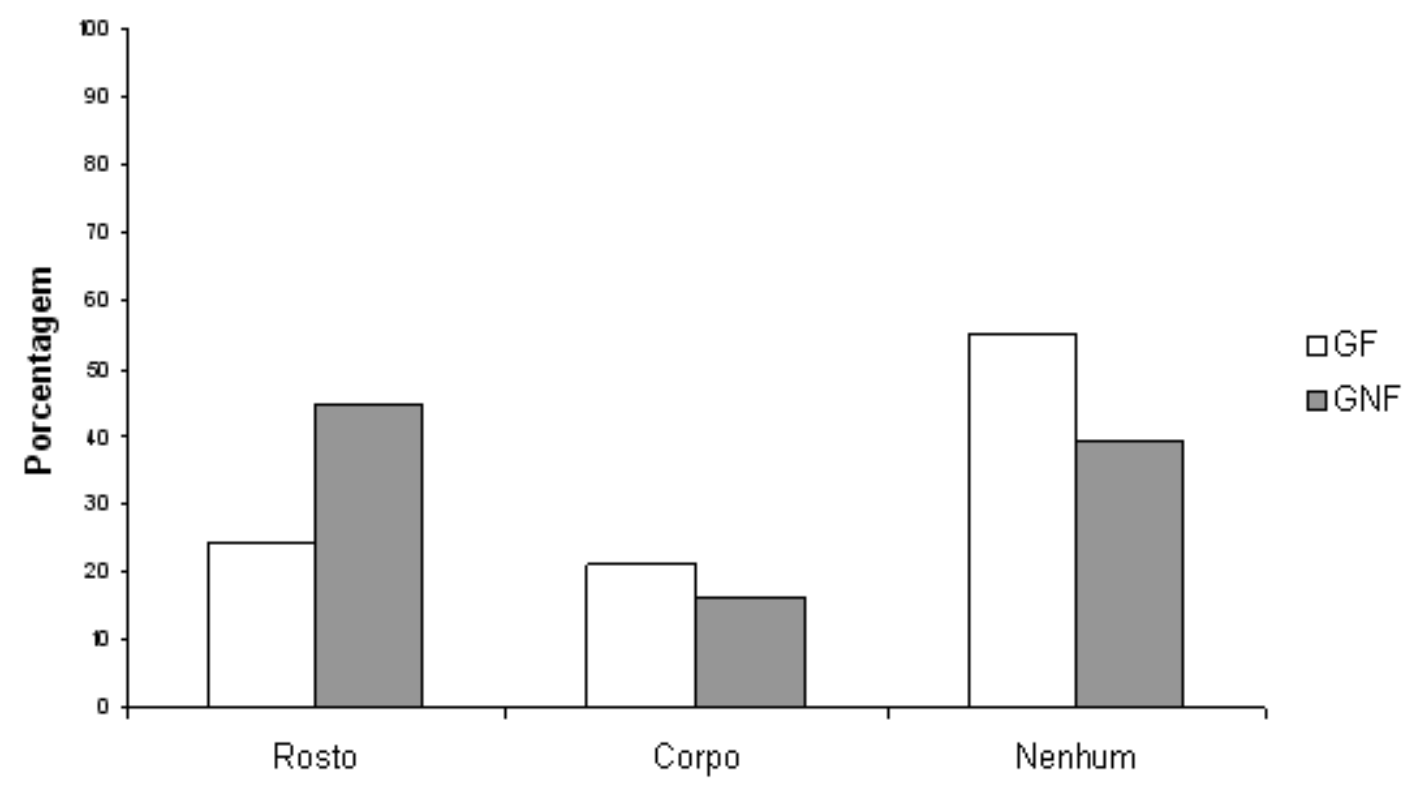

Aspectos

Figura 3. Aspectos fisicos que consideram importantes no outro para os participantes dos Grupos GF e GNF. 
grupos, elegendo a orelha. Entretanto, observa-se que o Grupo GNF valorizou mais a boca (19\%) e os dentes (18\%) do que o Grupo GF (respectivamente $12 \%$ e $15 \%$ ), dados que parecem confirmar a influência da fissura, corroborando as expectativas estabelecidas pela Teoria da Dissonância Cognitiva (Festinger, 1962/1975).

O teste estatístico de qui-quadrado realizado entre os grupos não evidenciou diferença significativa $\left(\mathrm{X}^{2}=4,633 ; p=0,5917\right)$ indicando similaridade entre os aspectos considerados importantes em geral. Sobre os aspectos do corpo que consideram importantes para o relacionamento afetivo, os resultados dos dois grupos são apresentados na Figura 4.

Quanto aos aspectos do corpo predominou, para os grupos, que não consideram nenhum aspecto importante, sendo $46 \%$ para o GF e $32 \%$ para o GNF, mas, as pernas, mais do que quaisquer outras partes do corpo ganham um destaque para $20 \%$ dos participantes do GF e $22 \%$ do GNF. O teste de quiquadrado aplicado entre os grupos, considerando-se todas as categorias de resposta, não evidenciou diferença significativa $\left(\mathrm{X}^{2}=7,263 ; p=0,2018\right)$.
Mais uma vez esta questão mostra a pouca importância dada pelos adolescentes pesquisados para o aspecto físico, com predominância do item "nenhum" nas respostas. Verifica-se, entretanto, uma diferença considerável nas respostas entre fissurados e não fissurados. Dessa forma, evidencia-se uma tentativa de redução da dissonância cognitiva (Festinger, 1962/1975), no GF.

Nas figuras a seguir, apresentam-se as respostas dos participantes às questões abertas sobre o que fazem quando estão paquerando alguém e o que esperam que o outro faça. As respostas foram inicialmente analisadas e agrupadas nas categorias descritas.

A Figura 5 mostra a freqüência de respostas, em porcentagem, para as categorias elaboradas a partir das respostas à pergunta "O que faz quando está paquerando alguém?". Observa-se que a categoria mais freqüente, para os dois grupos (GF e GNF) foi utilização do olhar, sendo $27 \%$ dos relatos do GNF e $20 \%$ do GF. Respostas como "Olho, sem me aproximar", "Troco olhares", "Fico olhando

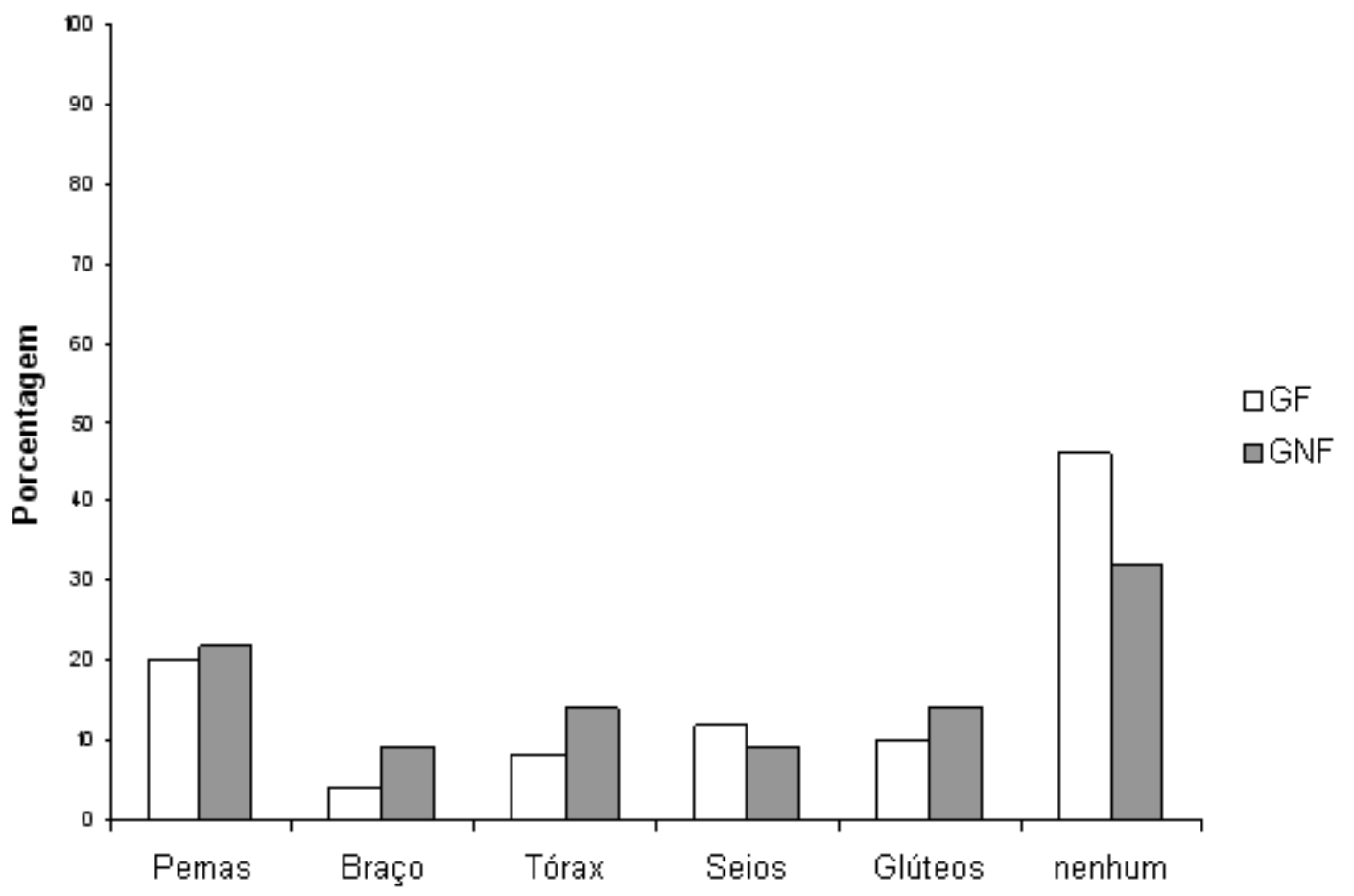

Figura 4. Aspectos do corpo que consideram importantes no outro, para os participantes dos Grupos Fissurados (GF) e Não Fissurados (GNF). 
até o outro perceber”, foram algumas dadas a essa questão.

Essa questão confirma os dados relativos aos aspectos do rosto, sendo os olhos considerados sua parte mais importante na paquera, para os dois grupos. Isso evidencia que os adolescentes, apesar de estarem em uma etapa da vida onde o belo parece ser muito valorizado, utilizam comportamentos observados e descritos por Fisher (1995) como o mais utilizado na hora do flerte, a comunicação não verbal. Para esta autora a dimensão não verbal é evidente, mesmo que inconsciente para o indivíduo. Dentro dessa forma de comunicação, o instrumento mais utilizado na paquera é a dinâmica do olhar. Como já foi dito anteriormente, esse comportamento desperta uma parte primitiva do cérebro causando a reação da rejeição ou aceitação (Fisher, 1995), imprescindível para a aproximação de pares. Silva (1991) reforça esta afirmação dizendo que o "olho no olho" funciona como sinal de querer estabelecer uma relação mais duradoura.

Outras respostas como "Faço o possível para que a pessoa se sinta feliz ao meu lado", "Se for um bom rapaz do tipo que me agrada, então vale a pena tentar derrubar todas as barreiras por ele", demonstram que nem todos os adolescentes estão preocupados apenas com o momento, mostrando-se preocupados com o futuro, se importando, também, com o caráter e a personalidade de seu pretendente, como pode ser visto também nas seguintes respostas: "Seja educado", "Que seja honesta, que tenha caráter", "Que seja legal, simpático", entre outras. Tais resultados coincidem com os apresentados na Figura 1 na qual se evidencia a importância de características psicológicas e sociais.

As categorias elencadas para a questão "o que você espera que o outro faça se quiser paquerar você?", estão apresentadas na Figura 6.

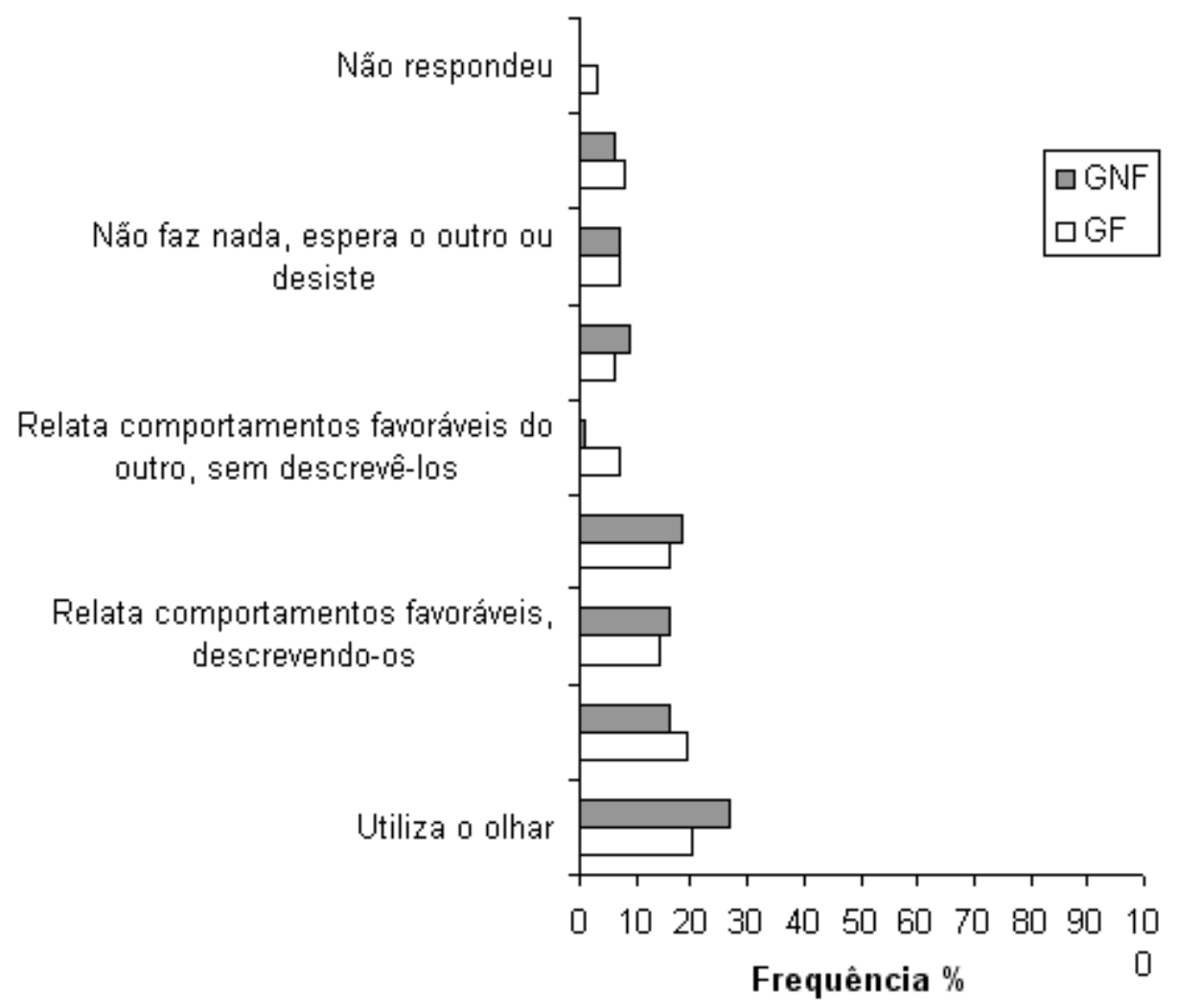

Figura 5. Comportamentos na paquera relatados pelos participantes dos Grupos Fissurados (GF) e Não Fissurados (GNF). 
Para esta questão as respostas dadas pelos sujeitos praticamente não diferem para os dois grupos. Para 38\% do Grupo GNF e para 36\% do Grupo GF os relatos dos participantes foram categorizados como "características desejáveis no outro" com frases do tipo: "Espero que seja sincero, honesto; que seja simpático”. Na categoria “comportamentos objetivos que espera do outro com relação a ele na hora de paquerá-1o", o Grupo GNF apresentou 36\% dos relatos e o Grupo GF, 31\%, com frases do tipo: "Espero que a pessoa retribua os olhares, espero que procure conversa". Na categoria "comportamentos subjetivos que espera do outro com relação a ele na hora de paquerá-lo", o Grupo GNF apresentou $27 \%$ dos relatos e o Grupo GF, 29\%, com frases como: "Espero que o outro se mostre interessado, que está a fim, espero que chegue até mim, que me aceite do jeito que sou".

Essas respostas comprovam o princípio da admiração descrito por Silva (2001), para quem para se apaixonar é preciso admirar. Nas respostas acima se pode ver bem isso, os adolescentes esperam encontrar traços que demonstrem admiração pelo outro, que completem suas expectativas pessoais, que correspondam aos seus interesses. Aron e Aron (1996) argumentam que quando uma pessoa é admirada, isso significa que possui qualidades que o admirador valoriza e gostaria de ter.

\section{Conclusão}

O presente trabalho identificou os critérios de seleção de parceiros afetivos em um grupo de adolescentes fissurados, comparando-os aos critérios utilizados por adolescentes não fissurados. Os dados obtidos mostraram que os dois grupos não apresentaram diferenças estatísticas entre si, apontando a honestidade e caráter como as características mais valorizadas e dinheiro e força física como as menos valorizadas. Em relação às outras características houve diferenças quantitativas, verificando-se diferença estatisticamente significativa ao se considerar aspectos corporais gerais (rosto, corpo ou nenhum).

A literatura da área tem mostrado que as pessoas tendem a escolher parceiros que têm as mesmas características valorizadas em si mesmas (homogamia). Para os dois grupos houve correlação significativa entre o que os participantes dos dois grupos disseram ser importante nele e no possível parceiro afetivo, de acordo, portanto, com a literatura.

As diferenças entre os grupos apareceram quando se perguntou qual aspecto físico (rosto, corpo ou nenhum) era considerado mais importante na paquera. Os dados evidenciaram diferença significativa entre os grupos, com maior valorização do rosto pelo GNF e do corpo pelo GF.

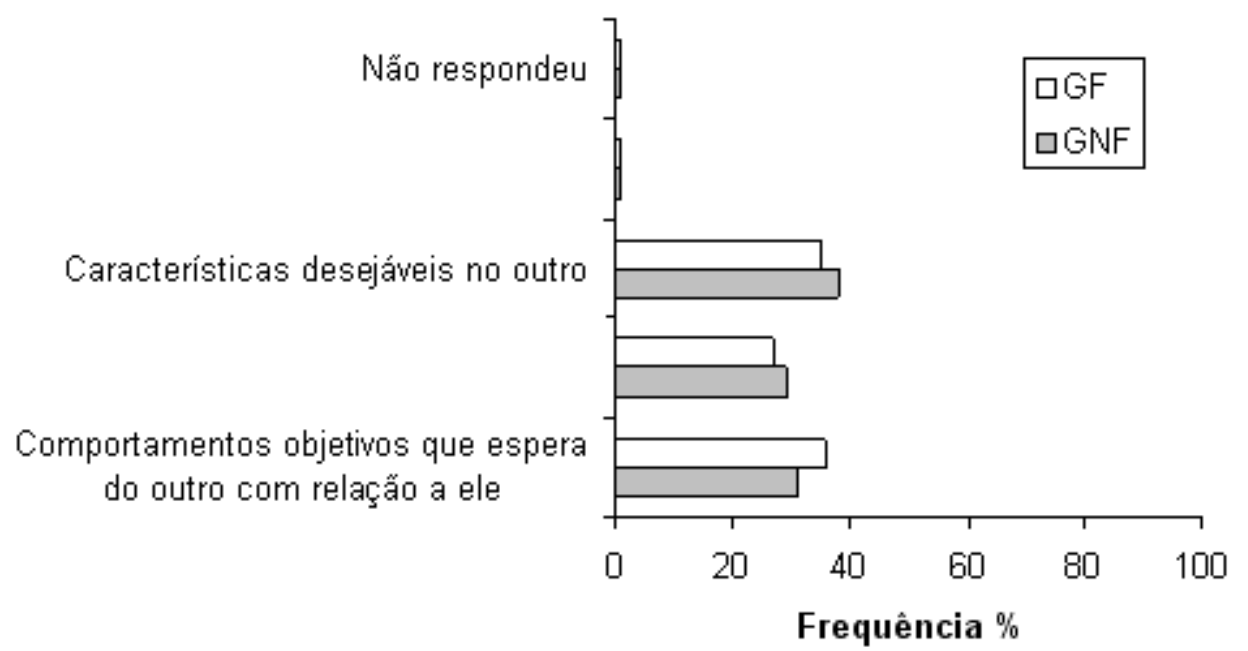

Figura 6. Comportamentos na paquera esperados pelos participantes dos Grupos Fissurados (GF) e Não Fissurados (GNF). 
No que diz respeito a aspectos do rosto, apesar dos dois grupos se referirem com mais freqüência aos olhos, o Grupo GNF apresentou percentuais maiores ao identificar a boca e os dentes como importantes do que o Grupo GF. Uma análise dessas respostas mostra que não há diferenças estatísticas significativas entre os grupos, permitindo concluir que a fissura interfere parcialmente na visão de si mesmo ou do outro na busca por parceiros afetivos. Com relação aos comportamentos relatados e esperados do outro na situação de paquera, os dois grupos não apresentaram categorias muito diferentes de respostas.

Os dados apresentados nesta pesquisa possibilitam uma visão parcial dos aspectos envolvidos na percepção corporal e auto-estima apresentados por portadores de fissura labiopalatal. Tais informações podem ser importantes no que diz respeito à condução de estratégias de tratamento integrado dos pacientes.

\section{Referências}

Aberastury, A. (1990). Adolescência. Porto Alegre: Artes Médicas.

Amaral, V. L. A. R. (1995). Aspectos psicológicos, problemas sociais e familiares associados às fissuras labiopalatinas. In S. Carreirão, S. Lessa, \& S. A. Zanini (Orgs.), Tratamento das fissuras labiopalatinas (pp. 19-30). Rio de Janeiro: Revinter.

Aron, E. N., \& Aron, A. (1996). Love and expansion of the self: The state of the model. Personal Relationships, 3, 45-58.

Bachega, M.I. (2002). Indicadores psicossociais e repercussões na qualidade de vida de adolescentes com fissura labiopalatal. Tese de Doutorado não-publicada, Universidade Estadual Paulista, Botucatu, SP.

Bee, H. (1996). A criança em desenvolvimento (7a ed.). Porto Alegre: Artes Médicas.

Blackwell, D. L., \& Lichter, D.T. (2004). Homogamy among dating, cohabiting, and married couples. The Sociological Quarterly, 45, 719-737.
Buston, P. M., \& Emlen, S. T. (2003). Cognitive processes underlying human mate choice: The relationship between self-perception and mate preference in Western society. Proceedings of the National Academy of Sciences of the United States of America, 100, 8805-8810.

Celen, N.; Cok, F., \& Bosma, H. A. (2006). A percepção de adolescentes e de pais sobre decisão e autonomia. Paidéia-Cadernos de Psicologia e Educação, 16, 349-363.

Chaves, J.C. (2001). “Ficar com”: Um novo código entre jovens. Rio de Janeiro: Revan.

Contini, M. L. J. (2002). Adolescência e psicologia: Concepções, práticas e reflexões críticas. Brasília, DF: Conselho Federal de Psicologia.

Davis, F. (1979). Comunicação não-verbal. São Paulo: Summus.

Duke, L. (2002) Get real! Cultural relevance and resistance to the mediated feminine ideal. Psychology and Marketing, 19, 211-233.

Erkolahti, R. K., Ilonen, T., \& Saarijarvi, S. (2003). Self-image of adolescents with diabetes mellitus type-I and rheumatoid arthritis. Nordic Journal of Psychiatry, 57, 309-312.

Festinger, L. (1975). Teoria da dissonância cognitiva. Rio de Janeiro: Zahar. (Original publicado em 1962).

Fisher, H. (1995). Anatomia do amor: A história natural da monogamia, do adultério e do divórcio. Rio de Janeiro: Eureka.

Goffman, E. (1988). Estigma: Notas sobre a manipulação da identidade deteriorada (4a ed.). Rio de Janeiro: Guanabara.

Gomes, G. R., \& Caramaschi, S. (2007). Valorização de beleza e inteligência por adolescentes de diferentes classes sociais. Psicologia em Estudo, 12, 295-303.

Hintikka, U., Pelkonen, M., Hintikka, J., Laukkanen, E., \& Lehtonen, J. (2002). Gender-specific differences in cognitive functioning and self-image among admitted adolescent psychiatric inpatients. Psychiatria Fennica, 33, 51-68. 
Jones, B. C., Litle, A. C., Boothroyd, L. G., Feinberg, D. R., Cornwell, R. E, DeBruine, L. M., Roberts, S. C., Penton-Voake, I. S., Smith, M. J. L., Moore, F. R., Davis, H. P., \& Perret, D. I. (2005). Women's physical and psychological condition independently predict their preference for apparent health in faces. Evolution and Human Behavior, 26, 451-457.

Kenrick, D. T., Sadalla, E. K., Groth, G., \& Trost, M. R. (1990). Evolution, traits and the stage of human courtship: Qualifying the parental investment model. Journal of Personality, 58, 97-116.

Lazar, A., \& Guttmann, J. (2004). Adolescents' perception of the ideal mate: Its relationship to parental characteristics in intact and nonintact families. Adolescence, 39, 389-396.

Li, N. P., \& Kenrick, D. T. (2006). Sex similarities and differences in preferences for short-term mates: What, whether and why. Journal of Personality and Social Psychology, 90, 468489.

Maner, J. K. (2003). Beauty captures the eye of the beholder: Selective attention and physical attractiveness. Dissertation Abstracts International, 64, 1551.

Michael, R. T., Gagnon, J. H., Laumann, E.O. \& Kolata, G. (1995). Sex in America: A definitive survey (hardcover). New York: Warner Books.

Pellegrini, A. D., \& Long, J. D. (2003). A sexual selection theory longitudinal analysis of sexual segregation and integration in early adolescence. Journal of Experimental Child Psychology, 85, 257-278.

Pratta, E. M. M., \& Santos, M. A. (2007). Opiniões dos adolescentes do ensino médio sobre o relacionamento familiar e seus planos para o futuro. Paidéia-Cadernos de Psicologia e Educação, 17, 103-114.

Regan, P. C., \& Joshi, A. (2003). Ideal partner preferences among adolescents. Social Behavior and Personality, 31, 13-20.
Rodrigues, J. L., Brino, R. F., \& Williams, L. C. A. (2006). Concepções de sexualidade entre adolescentes com e sem histórico de violência sexual. Paidéia-Cadernos de Psicologia e Educação, 16, 229-240.

Shackelford, T. K., Schmitt, D. P., \& Buss, D. M. (2005). Universal dimensions of human mate preferences. Personality and Individual Differences, 39, 447-458.

Silva, A. (1991). Rituais de flerte: A vez do olho no olho. Diálogo Médico, 17(4), 27-29.

Silva, A. A. (2001). O mapa do amor: Tudo o que você queria saber sobre o amor e ninguém sabia responder. São Paulo: Gente.

Tiba, I. (2005). Adolescentes: Quem ama, educa!. São Paulo: Integrare.

Weisfeld, G. E., \& Woodward, L. (2004). Current evolutionary perspectives on adolescent romantic relations and sexuality. Journal of the American Academy of Child and Adolescent Psychiatry, 43, 11-19.

Artigo recebido em 05/06/2007.

Aceito para publicação em 12/12/2007.

Endereço para correspondência:

Olga Maria Piazentin Rolim Rodrigues. Universidade Estadual Paulista Júlio de Mesquita Filho, Departamento de Psicologia. Av Engenheiro Edmundo Carrijo Coube, 1-40, Vargem Limpa. CEP: 17033-360, Bauru-SP, Brasil. E-mail: olgarolim@fc.unesp.br

Fernanda Renda Brasil é especialista em Psicologia Clínica pelo Hospital de Reabilitação de Anomalias Craniofaciais da Universidade de São Paulo, campus Bauru-SP.

Liliam D’Aquino Tavano é Doutora em Distúrbios da Comunicação pelo Hospital de Reabilitação de Anomalias Craniofaciais da Universidade de São Paulo, campus Bauru-SP, diretora técnica neste mesmo hospital. 
Sandro Caramaschi é Doutor em Psicologia Experimental pelo Instituto de Psicologia da Universidade de São Paulo, docente do Departamento de Psicologia da Universidade Estadual Paulista Júlio de Mesquita Filho, campus Bauru-SP.

Olga Maria Piazentin Rolim Rodrigues é Doutora em Psicologia Experimental pelo Instituto de Psicologia da Universidade de São Paulo, docente do Departamento de Psicologia da Universidade Estadual Paulista Júlio de Mesquita Filho, campus Bauru-SP. 